\title{
Preface to the IJAIED 25th Anniversary Issue
}

\author{
Monique Grandbastien ${ }^{1}$ • Rosemary Luckin ${ }^{2}$ • \\ Riichiro Mizoguchi ${ }^{3}$ - Vincent Aleven ${ }^{4}$
}

Published online: 19 January 2016

(C) International Artificial Intelligence in Education Society 2016

Keywords Artificial intelligence in education · Review - Overview

It is our great pleasure, as guest editors, to present an issue with invited articles by authors of high-impact articles from the journal's past. By the end of 2015, the International Journal of Artificial Intelligence in Education completed its 25 th volume and its 25 th year of operation. This milestone called for a celebration of a scholarly kind. The current issue is part of that celebration.

Although several landmark publications in the field appeared long before the journal's first year of publication (1989), the journal's early years coincided with the coalescing of the research community in Artificial Intelligence and Education (AIED). The same period saw the start of two relevant conferences, the International Conference on AI in Education (first held in 1987) and Intelligent Tutoring Systems (first held in 1988), although there had been two AI and Education conferences in the UK prior to

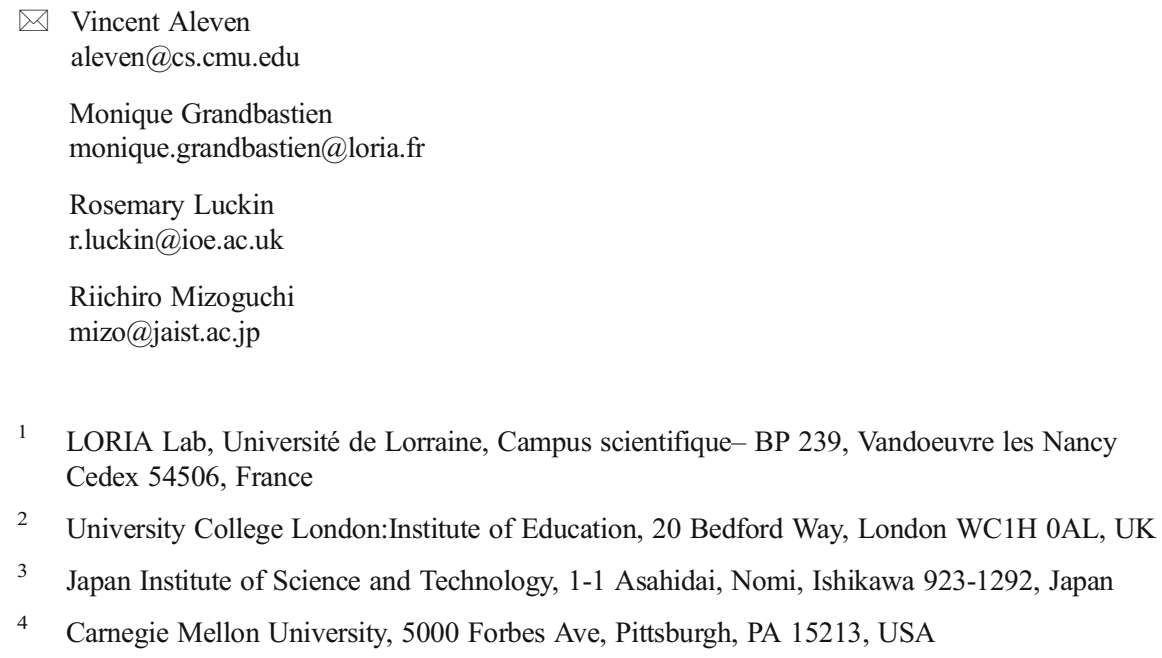

1 LORIA Lab, Université de Lorraine, Campus scientifique- BP 239, Vandoeuvre les Nancy Cedex 54506, France

2 University College London:Institute of Education, 20 Bedford Way, London WC1H 0AL, UK

3 Japan Institute of Science and Technology, 1-1 Asahidai, Nomi, Ishikawa 923-1292, Japan

4 Carnegie Mellon University, 5000 Forbes Ave, Pittsburgh, PA 15213, USA 
1987. From 1989 until 1995 the journal was titled the Journal of Artificial Intelligence in Education. Its name changed to the current one in 1995, though the volume numbers continued in sequence. A history of the International AIED Society, its conferences, and the journal is given in John Self's article "The Birth of IJAIED," included in this issue. Here is a good place to record our immense gratitude to John Self who steered the Society and the journal to its current form.

Over the first 25 years of its existence, the journal has been a vibrant forum publishing the best work on the use of AI methods to advance education. Approximately 400 articles have appeared in the journal. Google Scholar indicates that the median number of citations per article is about 20 , with an average number of citations of about 55. With the current growth in the use of educational technologies, and the concomitant rise of our research field, these numbers can be expected to increase.

Since a research field needs to have a sense of its history, we decided to compile an issue in which the authors of the journal's most impactful original research papers reflected on their own classic article. Specifically, we approached the authors of highlycited articles from the journal's history to write a new, short article that presented a new outlook on the line of work featured in their historic article, including subsequent developments and future directions. Rigorous scholarly standards demanded an objective definition of what it means to be highly-cited! We settled on 100 citations as listed in Google Scholar as our inclusion criterion.

We asked authors to address the following questions:

(1) Motivation: What was the core problem that motivated the work?

(2) Approach: What were the key drivers?

(3) Core contributions: What was the key intellectual contribution of this paper?

(4) Practical impact: What was the key practical contribution of this paper? In what way did the work in the paper facilitate deployment, in real educational settings, of AIED systems?

(5) Core limitations: What is still an open issue?

(6) Subsequent research: What progress has been made, by you or others, on the problem addressed in your paper since it came out?

(7) Future directions: What are the future needs for tackling the problem addressed in your paper?

We received the 35 articles presented in the current issue. All went through the regular stringent peer review process of the journal, many with multiple cycles of revisions. We asked the reviewers to evaluate not only whether the article met the regular review criteria, but also whether it addressed the seven questions above.

In writing this preface, as guest editors, we decided not to try to synthesize general trends and lessons learned from this set of articles, simply because that is too daunting a scholarly task. Instead, we would like to call attention to the scholarly depth and breadth of the articles received, as well as the remarkable efforts by the authors in eloquently reminding us of the conceptual and technical context in which their initial paper was written. We are struck, also, by the staying power of many of the featured projects, which often over many years reached a state of substantial maturity and produced sharable and reusable results, surviving — often capitalizing on — rapid changes in the underlying technologies used. Many of the projects are still going strong and 
have strong implications for future research. Our history is influencing us as we explore new and improved ways in which technology can impact education.

This issue comes at a time of renewed interest in AI generally and an increased interest in $\mathrm{AI}$ and education, with much talk about it in the media. For example, Andy Rubin, the creator of the Android platform, tells us that AI will be the next big development in computing: a new platform that will support significant positive applications across many sectors, including education. Investment in AI companies has been huge: in 2014 funding for AI start-ups increased by $302 \%$ \% ${ }^{1}$ Similarly, investments in educational technology companies are up. We envision considerable possibilities for AIED as it reaps the benefits of this upsurge in investment. The current issue can offer a foundation in the research that has grounded the field and help form the all-important links between research and application within the wider world.

We thank all authors of the articles in this issue. We also thank all reviewers, who at times must have felt that we were calling on them more frequently than in other years. And indeed we did, given the large number of papers that were submitted. We hope this issue will serve as a guide for those new to the field while also providing food for thought for those who already have a good overview or are steady contributors.

Here is to the next 25 years of artificial intelligence in education!

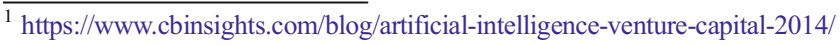

